\title{
A Survey Report on Channel Estimation for Compressive Sensed OFDM System under Long Delay Channel
}

\author{
Pratibha Yadav \\ M.Tech. Scholar \\ Department of Electronics \& \\ Communication Engineering, \\ Truba Institute of Engineering \\ and Information Technology \\ Bhopal, India
}

\author{
Neelesh Gupta, PhD \\ H.O.D Department of \\ Electronics \& Communication \\ Engineering, Truba Institute of \\ Engineering and Information \\ Technology Bhopal, \\ India
}

\author{
Neetu Sharma \\ Asst. Prof. Department of \\ Electronics \& Communication \\ Engineering, Truba Institute of \\ Engineering and Information \\ Technology Bhopal, \\ India
}

\begin{abstract}
Orthogonal frequency division multiplexing (OFDM) is particularly divided to three varieties. Cyclic prefix OFDM, zero padding OFDM, and time domain synchronous OFDM. Compared to CP OFDM, TDS-OFDM has higher spectral efficiency and faster synchronization. This paper reviewed however efficiently uses the compressive sensing (CS) theory to resolve those problems. During this paper, a noise cancelation scheme using compressed sensing (CS) technique is planned for orthogonal frequency division multiplexing systems. From the chosen reliable observations, the noise in time domain is reconstructed and canceled by using CS technique. TDS-OFDM has fractional channel priori, therefore by suggests that of this presentation be ready to improved and reduce the complication of the algorithmic rule.
\end{abstract}

\section{Keywords}

TDSOFDM, mutual interferences, compressive sensing (CS), Channel estimation (CE), long delays, OFDM

\section{INTRODUCTION}

OFDM may be a signal technique that has been applied usually within the wireless communication systems by reason of its ability to require care of the effective transmission and highly economical bandwidth utilization that's inside the presence of various channel impairments one amongst them may be a frequency discerning fading. Within the OFDM systems existing spectrum are divided into the many orthogonal and sub-channels, that are instantly used within the data transmission. Throughout this method the inter-symbol interference (ISI) that's induced due to frequency discerning channels could also be reduced by adding the cyclic prefix (CP) [1]. Spectrum and energy efficiency are of great importance for present and future wireless communication systems [8]. OFDM has previously been extensively implemented by many wireless communication ways like DVB-T, WiMAX, LTE, WiFi, etc, and it's additionally widely known as a prominent modulation technique for future wireless communication systems [9]. Thus, developing spectrum- and energy-efficient OFDM scheme is important to realize high transmission efficiency and low energy consumption. There are three basic kinds of OFDM: cyclic prefix OFDM [9], zero padding OFDM [10], and time domain synchronous OFDM [11]. The accepted CP-OFDM makes use of a $\mathrm{CP}$ as a guard interval to alleviate inter-block-interference (IBI) in multipath channels [7]. On the opposite hand, in TDSOFDM, the IBI between the TS and also the OFDM information block must be cancelled by the unvarying padding subtraction (IPS) methodology that involves the mutually conditional channel estimation and channel deed. During this paper, to boost the system performance of TDSOFDM over quick time-varying channels, we tend to propose a unique time-frequency signal structure and also the corresponding joint time-frequency channel estimation methodology to accurately track the fast variation of the wireless fading channel. The application of Cs techniques to the matter of channel estimation was initial planned in 2008, and since then many similar channel estimators are introduced, that we tend to all subsume under the name of compressive channel estimation. they're supported the very fact that in typical wireless transmission situations the signal takes many various ways from the transmitter to the receiver (for example owing to reflections from large objects like homes, trees, cars, mountains, etc.), however that always the number of such multipath elements yielding important contributions at the receiver is very restricted. The smallest amount sq. and minimum mean-square error (MMSE) is predicted linear channel illation technique that relies on the pilot arrangement. The LS method is a smaller amount complicated and easy regard to the opposite ways and consequently is employed to channel estimation; however it's a significant weakness that's a lot of attentive to the channel noise. Hsieh used a comb style of the pilot arrangement and second order interpolation technique to the channel estimation [7]. Coleri et al. compared to the results of the many interpolation technique channel estimation with the Rayleigh fading like linear, second order, cubic, low pass filtering and spline interpolation ways the recently introduced principle and methodology of compressed sensing (CS) permits the efficient reconstruction of sparse signals from very limited variety of measurements (samples). The motivation to use compressive sensing in channel estimation is that the observation that some channels are characterized by sparse multipath - by that we tend to mean that there are a lot of fewer distinct arrivals as there are baseband channel taps. With this in mind compressive sensing promises to estimate the channel with a lot of fewer pilots overhead or at higher accuracy with a constant variety of pilots. During this work, channel estimation in OFDM systems is investigated. the most objective of this thesis is to investigate the performance of channel estimation in OFDM systems and study different patterns of pilot symbols that already have been planned in literature. Contributions of this paper are 1st, cancelling the interferences if present, and use the concept of inter-block- 
interference (IBI) - free region of small size to reconstruct the high-dimensional multipath channel. There are several benefits and downsides to OFDM. Benefits are, Immunity to delay spread \&amp; multipath, makes efficient use of the spectrum by permitting overlap, is less sensitive to sample timing offsets than single carrier systems are. As several authors take a closer look at this and realize that in a very channel modeled by specular (point) scatterers the amount of nonzero baseband taps depends very much on what one defines as negligible. Using instead an oversampled baseband model, the illustration of the channel becomes ambiguous, however additionally sparser. Cs permits sparse signals to be recovered from only a few measurements, which frequently translates to fewer samples and shorter sensing times.

\section{LITERATURE SURVEY}

Wenbo Ding et. al. [1] "Compressive Sensing Based Channel Estimation for OFDM Systems under Long Delay Channels" To solve this drawback, a channel estimation methodology for OFDM below the framework of compressive sensing (CS) is projected during this paper. Time-domain synchronous orthogonal frequency division multiplexing (TDS-OFDM) has benefits in spectral efficiency and synchronization. Firstly, by exploiting the signal structure of recently projected timefrequency training OFDM scheme, the auxiliary channel data is obtained. Secondly, we tend to propose the auxiliary data based subspace pursuit (A-SP) algorithmic rule to utilize a much bit of frequency domain pilots embedded within the OFDM block for the exact channel estimation. The MSE performance of this methodology outperforms the standard schemes and is close to the CRLB by simultaneously exploiting the time-domain PN sequence and frequencydomain pilots.

Shail Prakash Singh et. al. [2] "Overview of Compressive Sensing Based Channel Estimation for OFDM Systems under Long Delay Channels" In OFDM systems the out there spectrum are divided into several orthogonal sub-channels, that are instantaneously wont to information transmission. In OFDM systems, channel estimation is important to get the channel state data (CSI), reducing the bit error rate and additionally to achieve a distortion less output information. The least square and minimum mean-square error (MMSE) are standard linear channel estimation techniques that are supported pilot arrangement. The MSE performance of this methodology outperforms the standard schemes and is near the CRLB by at the same time exploiting the time-domain PN sequence and frequency-domain pilots. during this paper planned a CS-based cerium methodology for OFDM by using the auxiliary channel data, the planned SP algorithmic rule has lower complexness than the standard SP algorithmic rule.

Wenbo Ding et. al. [3] "Simultaneous Time-Frequency Channel Estimation Based on Compressive Sensing for OFDM System" In this letter, a simultaneous time frequency channel estimation technique supported compressive sensing (CS) is planned to resolve this downside. Besides, the auxiliary channel data is used to additional reduces the complexness of the classical CS algorithmic rule. The planned simultaneous time-frequency CE technique supported CS for TDS-OFDM will support the high-order modulations like 256QAM under severe fading channels once the most delay is fairly near the GI length. Thus, the planned technique is expected to increase TDS-OFDM within the emerging UHDTV applications.

Kee-Hoon Kim et. al. [4] "Clipping Noise Cancelation for OFDM Systems Using Reliable Observations Based on
Compressed Sensing" In this paper, a clipping noise cancelation scheme using compressed sensing (CS) technique is planned for orthogonal frequency division multiplexing systems. Instead, observations of the clipping noise in information tones are exploited, that results in no loss of information rate. From the chosen reliable observations, the clipping noise in time domain is reconstructed and canceled by using Cs technique. The planned scheme doesn't would like reserved tones or pilot tones that are different from the previous works using Cs technique. During this paper, a brand new clipping noise cancelation scheme using Cs for OFDM systems is planned. To reconstruct the clipping noise, the planned scheme exploits its compressed observations underlying within the information tones less contaminated by channel noise. The planned clipping noise cancelation scheme cancels out the clipping noise overflow an AWGN channel and a frequency-selective fading channel that is verified through simulations. During this letter, a spectrum-efficient MIMO-TFT-OFDM various to the quality MIMO-OFDM scheme is planned by planning a time-frequency joint sparse channels estimation with high accuracy under the framework of SCS.

Linglong Dai et. al. [5] "Joint Time-Frequency Channel Estimation for Time Domain Synchronous OFDM Systems" In this paper, a unique time-frequency signal structure is planned to enhance the system performance of TDS-OFDM over fast time-varying channels. The modularly orthogonal sequence (MOS) with excellent autocorrelation property is adopted because the TS, whereas every pilot group has only 1 non-zero central pilot surrounded by many zero pilots. During this paper, authors have investigated a unique time-frequency signal structure and therefore the corresponding joint timefrequency channel estimation methodology to enhance the performance of TDS-OFDM systems over fast fading channels. It's been demonstrated that the planned methodology might accurately track the channel variation, and outperforms the standard solutions over fast time-varying channels.

\section{METHOD}

For with success accomplish any thesis, there ought to be a well-planned approach to achieve the will goal. Orthogonal frequency division multiplexing (OFDM) techniques is mentioned during this chapter. This chapter includes the method utilized in the thesis. This chapter additionally includes diagram and technique used for orthogonal frequency division multiplexing (OFDM) system.

Compressive Sensing Orthogonal Frequency Division Multiplexing (CS-OFDM) developed by Cisco, is an open normal for broadband wireless net services. CS-OFDM will increase subscriber coverage for high-speed, high-reliability net, packet local and long distance telephone services and Virtual private Network (VPN) access. CSOFDM additionally lowers the price of provisioning and deploying infrastructure for a wireless network, and utilizes multipath signals to enhance or recreate transmitted signals, dramatically increasing overall system performance and scalability for service providers. This enables for high data throughput during a minimum of radio frequency (RF) spectrum and supports extreme multipath problems existing in obstructed or part obstructed non-line-of-sight environments. Just like standard OFDM, in CS-OFDM, the modulated symbols are processed block-by-block. Assume that there are $\mathrm{N}=\mathrm{LM}$ modulated symbols in one block. 


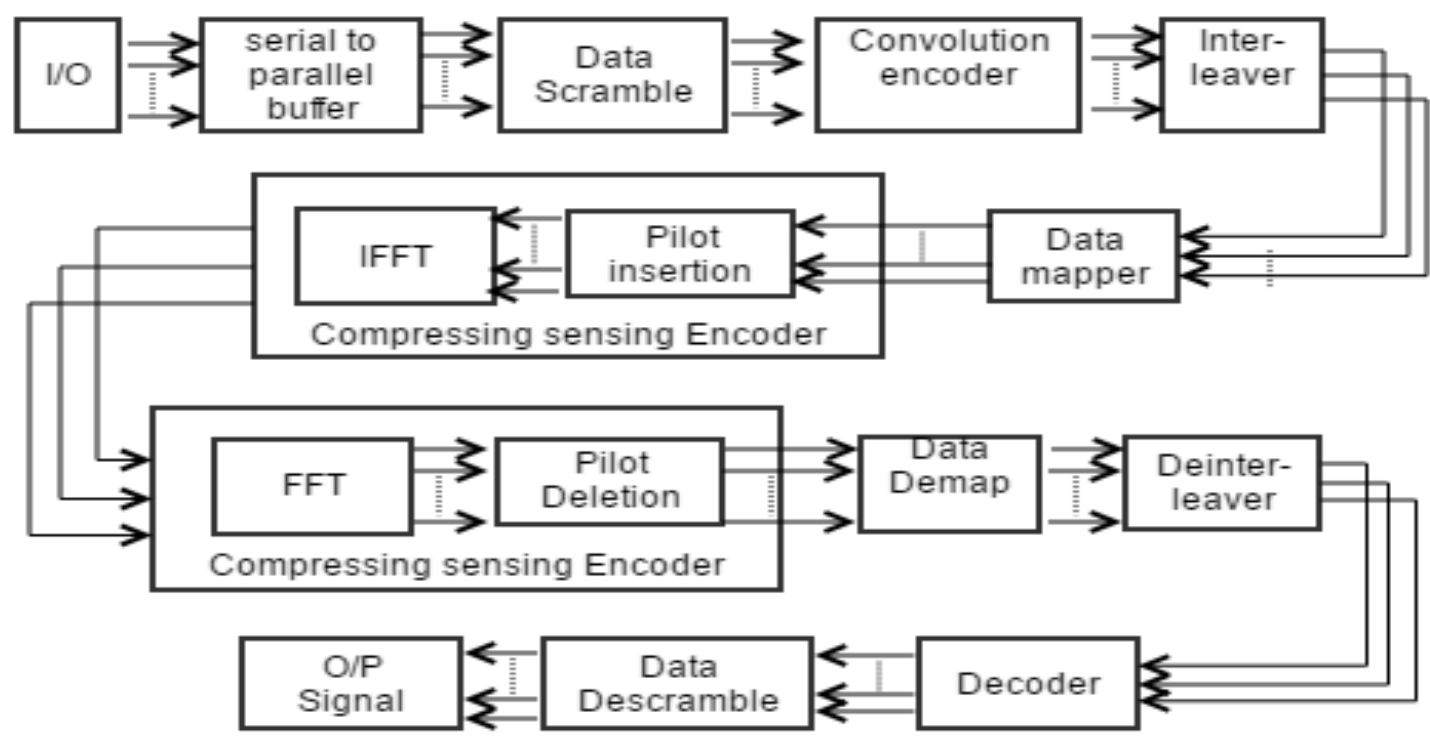

Figure 1: Block diagram of Compressive Sensing Orthogonal Frequency Division Multiplexing (CS-OFDM

\subsection{Attenuation}

Attenuation is that the drop in the signal power once transmission from one purpose to a unique. it'll be caused by the transmission path length, obstructions in the signal path, and multipath effects. Any objects that obstruct the line of sight of the signal from the transmitter to the receiver will cause attenuation. Shadowing of the signal can occur whenever there is an obstruction between the transmitter and receiver. It is mostly caused by buildings and hills, and is the most important environmental attenuation issue. Shadowing is that the most severe in heavily designed up areas, as a results of the shadowing from buildings.

\subsection{Multipath Effects on Rayleigh Fading}

In a radio link, the RF signal from the transmitter perhaps reflected from objects like hills, buildings, or vehicles. This offers rise to multiple transmission strategies at the receiver. Figure 2 shows a number of the possible ways that in which multipath signals will occur. The relative phase of multiple reflected signals will cause constructive or destructive interference at the receiver. This can be experienced over very short distances (typically at 0.5 wavelength distances), that's given the term fast fading.

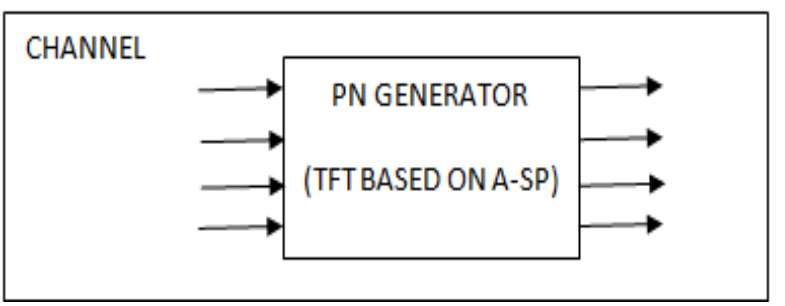

Figure 2: Box diagram representation of PN generator

\subsection{Frequency Selective Fading}

In any radio transmission, the channel spectral response is not flat. it's dips or fades inside the response due to reflections causing cancellation of certain frequencies at the receiver. Reflections off near-by objects (e.g. Ground, buildings, trees, etc) can result in multipath signals of similar signal power because the direct signal. This may lead to deep nulls inside the received signal power due to destructive interference. For narrow bandwidth transmissions if the null in the frequency response happens at the transmission frequency then the entire signal will be lost.

\subsection{Delay Spread}

The received radio signal from a transmitter consists of usually a direct signal and signals reflected off object such as buildings, mountains, and different structures. The reflected signals arrive at a later time than the direct signal as a result of the additional path length, giving rise to a rather totally different time of arrival of the transmitted pulse. The signal energy confined to a narrow pulse is spreading over a extended time. Delay spread may be a measure of but the signal power is spread over the time between the arrival of the primary and last multipath signal seen by the receiver. During a digital system, the delay spread can result in inter-symbol interference.

\section{CONCLUSION}

In this paper we tend to reviewed Compressive Sensing primarily based Channel Estimation for OFDM Systems under Long Delay Channels and related work. In [1] projected a CS-based CE methodology for TFT-OFDM. Projected scheme features a good BER performance in each static and mobile situation and might well support the 256 QAM, particularly once the maximum channel delay spread is fairly near or maybe larger than the GI length. In [2] projected a CS-based cerium methodology for OFDM during this paper. The MSE performance of this methodology outperforms the traditional schemes and is near the CRLB by simultaneously exploiting the time-domain PN sequence and frequencydomain pilots.

\section{REFERENCES}

[1] Ding, Wenbo, Fang Yang, Changyong Pan, Linglong Dai, and Jian Song. "Compressive sensing based channel estimation for OFDM systems under long delays channels" IEEE Trans. on Broad-casting 60, no. 2 (2014): 313-321. 
[2] Singh, Shail Prakash, and Rashmi Pandey. "Overview of Compressive Sensing Based Channel Estimation for OFDM Systems under Long Delay Channels" (2012).

[3] Ding, Wenbo, Fang Yang, Chao Zhang, Linglong Dai, and Jian Song. "Simultaneous time-frequency channel estimation based on compressive sensing for OFDM system" In Global Commu. Conference 2014 IEEE, pp. 3573-3578. IEEE, 2014.

[4] Kim, Kee-Hoon, Hosung Park, Jong-Seon No, Habong Chung, and Dong-Joon Shin. "Clipping noise cancelation for OFDM systems using reliable observations based on compressed sensing" IEEE Transactions on Broadcasting 61, no. 1 (2015): 111-118.

[5] Dai, Linglong, Zhaocheng Wang, Jun Wang, and Zhixing Yang. "Joint time-frequency channel estimation for time domain synchronous OFDM systems" IEEE Trans. on Broadcasting 59, no. 1 (2013): 168-173.

[6] L. He, F. Yang, C. Zhang, and Z. Wang, "Synchronization for TDSOFDM over multipath fading channels" IEEE Trans. Consum. Electron. vol. 56, no. 4, pp. 2141-2147, Nov. 2010.

[7] J. Song, Z. Yang, L. Yang, K. Gong, C. Pan, J. Wang, and $\mathrm{Y}$. Wu, "Technical review on Chinese digital terrestrial television broadcasting standard and measurements on some working modes" IEEE Trans. Broadcast., vol. 53, no. 1, pp. 1-7, Feb. 2007.

[8] J. Wang, Z. Yang, C. Pan, and J. Song, "Iterative padding subtraction of the PN sequence for the TDS-
OFDM over broadcast channels," IEEE Trans. Consum. Electron., vol. 51, no. 11, pp. 1148-1152, Nov. 2005.

[9] S. lk Park, G. Lee, Y. Wu, H. M. Kim, N. Hur, J. H. Chung, and J. Kim, "Backward compatible UHDTV transmission based on ADT system for the ATSC terrestrial DTV broadcasting" in Proc. IEEE Int. Symp. Broadband Multimedia Syst. Broadcast, Jun. 2013.

[10] J. Fu, J. Wang, J. Song, C. Pan, and Z. Yang, "A simplified equalization method for dual $\mathrm{PN}$-sequence padding TDS-OFDM systems" IEEE Trans. Broadcast., vol. 54, no. 4, pp. 825-830, Dec. 2008.

[11] L. Dai, Z. Wang, and Z. Yang, "Compressive sensing based time domain synchronous OFDM transmission for vehicular communications" IEEE J. Sel. Areas Commun., vol. 31, no. 9, pp. 460-469, Sep. 2013.

[12] K. Yan, F. Yang, C. Pan, and J. Song, "Reception quality prediction in a single frequency network for the DTMB standard" IEEE Trans. Broadcast., vol. 58, no. 4, pp 629-636, Dec. 2012.

[13] J. Wang, J. Song, J. Wang, C. Pan, Z. Yang, and L. Yang, "A general SFN structure with transmit diversity for TDS-OFDM system" IEEE Trans. Broadcast., vol. 52, no. 2, pp. 245-251, Jun. 2006

[14] L. Dai, Z. Wang, and Z. Yang, "Time-frequency training OFDM with high spectral efficiency and reliable performance in high speed environments" IEEE J. Sel. Areas Commun., vol. 30, no. 4, pp. 695-707, May 2012. 